C-A/AP/\#335

November 2008

\title{
"Matching" the BtA line to the bare-AGS (Part 1)
}

\author{
N. Tsoupas, J.W. Glenn, H. Huang, W.W. MacKay, D. Raparia, K. Zeno
}

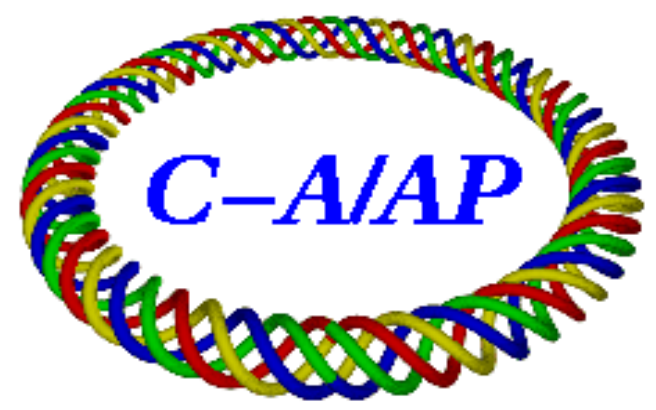

\section{Collider-Accelerator Department Brookhaven National Laboratory Upton, NY 11973}

Notice: This document has been authorized by employees of Brookhaven Science Associates, LLC under Contract No. DE-AC02-98CH10886 with the U.S. Department of Energy. The United States Government retains a nonexclusive, paid-up, irrevocable, world-wide license to publish or reproduce the published form of this document, or allow others to do so, for United States Government purposes. 


\title{
"Matching" the BtA line to the bare-AGS (Part I)
}

\author{
N. Tsoupas, J. W. Glenn, H. Huang, W.W. MacKay, D. Raparia, K. Zeno
}

\section{Abstract}

The Booster to AGS (BtA) transfer line [Ref for BtA line ] transports the beam bunches from the AGS-Booster to the AGS synchrotron, and also matches the beam parameters

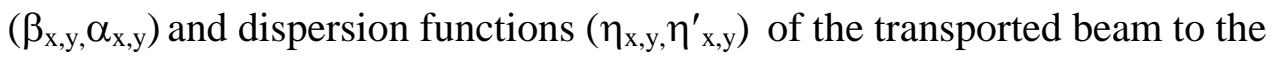
corresponding quantities of the circulating beam in AGS, at the AGS injection point. In this technical note we describe in details, the calculations of the matching procedure of the BtA line to the bare-AGS, and provide magnet settings for the MAD-model of the BtA transfer line which is "matched" to the bare-AGS. In a separate but more concise technical note (Part II) we will present results on the beam optics of the BtA beam line which is "matched" to the AGS with two helical snakes.

\section{Introduction}

The BtA transfer line transports beam bunches from the AGS-Booster to AGS synchrotron and also matches the beam parameters $\left(\beta_{x, y}, \alpha_{x, y}\right)$ and dispersion functions $\left(\eta_{x, y}, \eta_{x, y}^{\prime}\right)$ of the transported beam to the corresponding quantities of the circulating beam in AGS, at the AGS injection point. The above process, of beam transport and beam matching, will be referred to in this technical note as "BtA matching". Also, the AGS injection point in this note is defined as the middle of the straight section A06 of the AGS. Figure 1 is a schematic diagram of the relative locations of the AGS-Booster, the BtA line, and AGS. The same figure shows the relative locations of the various devices which are used to extract the beam bunches from the AGS-Booster and inject the beam into the AGS.

In this technical note we present in details the procedure we follow for the "BtA matching”. The procedure includes the following steps:

a) Beam extraction from the AGS-Booster and calculations of the beam parameters and dispersion functions, at the starting point of the BtA line, which, in this technical note is defined $15 \mathrm{~cm}$ from the end of the $\mathrm{F} 6$ extraction septum of the Booster.

b) Calculation of the beam parameters $\left(\beta_{x, y}, \alpha_{x, y}\right)$ and dispersion functions $\left(\eta_{x, y}, \eta_{x, y}^{\prime}\right)$ of the circulating beam in AGS at the middle of the straight section A06 (injection point) of AGS.

c) The computation of the $\mathrm{R}$ transfer matrix, between the end point of the BtA line (28.6 cm from the exit of QV15) and the middle of the A06 straight section (AGS injection point). This $\mathrm{R}$ matrix will be used in the matching of the BtA line and the AGS. 
d) Matching of the BtA line to the AGS at the middle of the straight section A06. In the matching procedure we use a MAD model of the BtA line and we also use the $\mathrm{R}$ matrix as calculated in step c) above. The beam parameters of the injected beam are matched to those of the circulating beam at the AGS injection point.

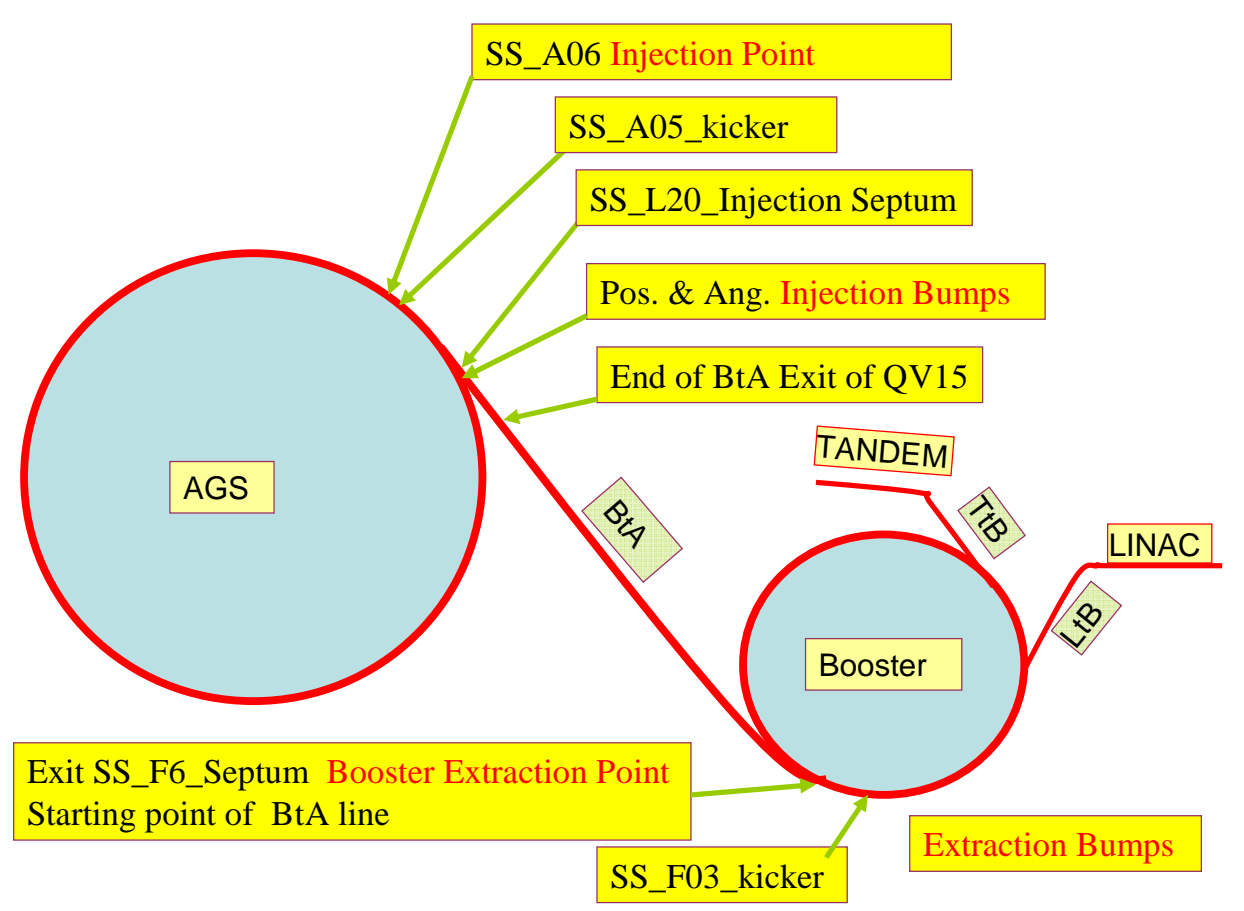

Figure 1. Schematic diagram of the AGS-Booster, the BtA line, and AGS synchrotron. The location of the Booster extraction devices, and the AGS injection devices are also shown.

\section{Beam Extraction from the AGS- Booster and calculations of the beam parameters at the starting point of the BtA line.}

In order to perform the the "BtA matching" the knowledge of certain physical quantities are required. One set of such quantities is the beam parameters and dispersion functions at the beginning of the BtA line.

In the following subsection we describe the procedure to calculate these quantities. In the calculations we use MAD models for both, the AGS-Booster synchrotron, and the Booster Extraction section. The Booster Extraction section is the section of the beam line between the F3 extraction kicker of the AGS-Booster, and the beginning of the BtA line.

\section{Set up the AGS-Booster at Extraction.}

Using a MAD model of the AGS Booster we set up the synchrotron to simulate the circulating beam just before beam extraction. The beta functions $\left(\beta_{\mathrm{x}, \mathrm{y}}\right)$ and dispersion functions $\left(\eta_{x, y}\right)$ of the AGS-Booster at extraction are plotted in Fig. 2 as a function of the location around the ring. A set of "back leg windings" which are mounted on specified main magnets, are excited to generate a local bump which displaces the circulating beam 
near the F6 extraction septum. Figure 3 shows the local beam bump (black solid line) at the section of the Booster where the F6 septum is located. The vertical green line in Fig. 3 indicates the location of the F6 extraction septum. The excitation of the extractionbump-magnets does not significantly affect the beta functions or the dispersion functions of the closed orbit. A more detailed description of the AGS-Booster extraction mechanism is presented in Ref. [2]. Figure 4 has been borrowed from Ref. [2] and shows the location of the main magnets with back leg windings, the F3 extraction kicker, and the F6 extraction septum. In the same figure, shown are, the closed orbit displacement just before beam extraction, and part of the extracted trajectory after the F3 extraction kicker is excited to extract the beam from the AGS Booster.

\section{$\beta_{x, y}$ functions at Booster Extraction}

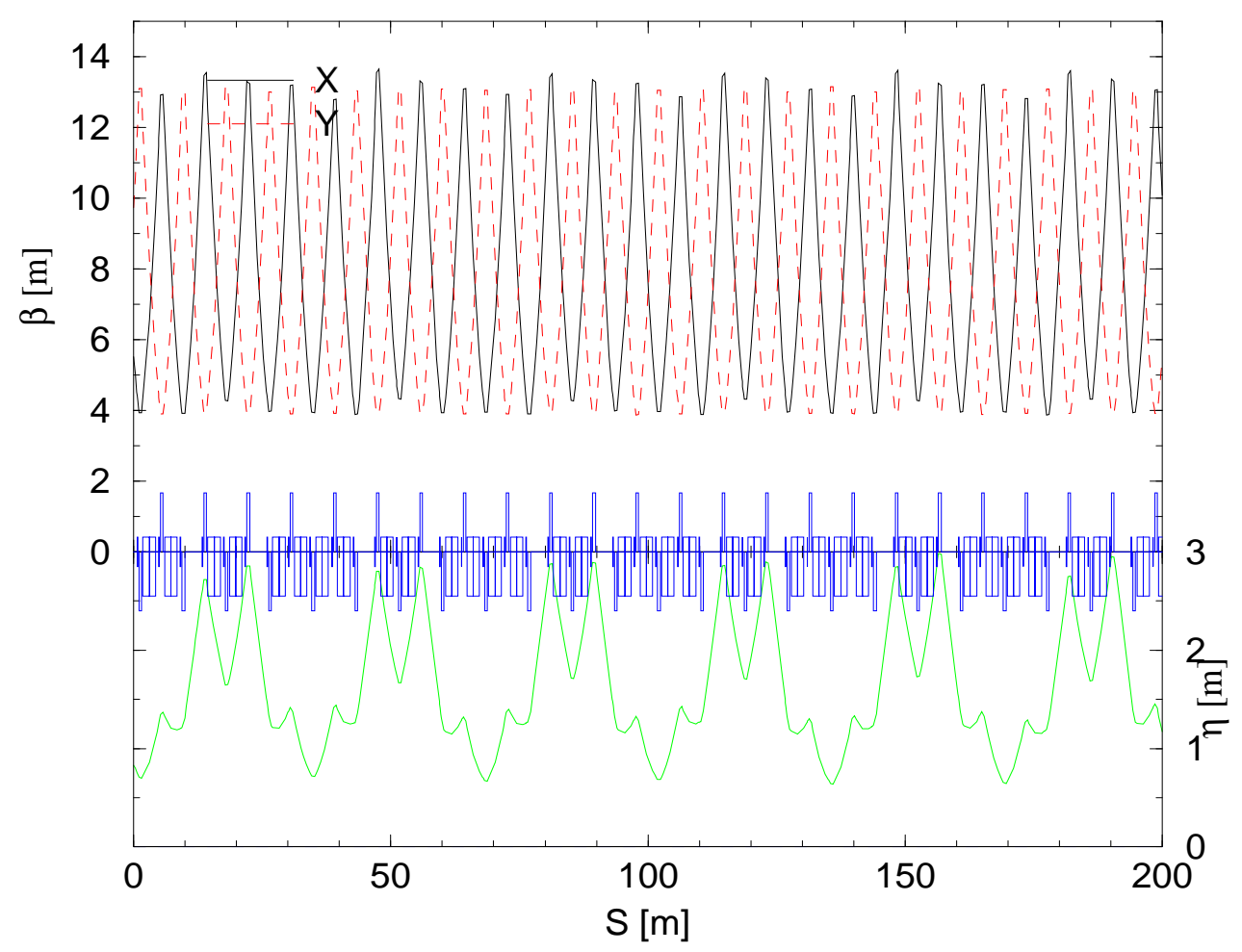

Figure 2. The Horizontal (black line) beta function, the Vertical (red dashed line) beta function and the horizontal dispersion function (green line) along the AGS-Booster just before beam extraction.

The bumped-circulating beam is extracted by the F3 kicker into the main field of the F6 septum which guides the beam at the "Booster extraction point". This point is also defined, in this technical note, as the "beginning of the BtA line". Figure 5 shows the beta and dispersion functions along the extraction section of the AGS-Booster, which starts at the F3 kicker and ends at the exit of the F6 septum. The trajectory of the extracted beam, in the extraction section of the AGS-Booster, is shown in Figure 6. The last two blue boxes in the figures 5 and 6 is the F6 septum magnet which is comprised of two dipoles. 
Table 1 shows the calculated beam parameters at the extraction point of the AGS-Booster for two different extraction settings of the AGS-Booster. From the comparison of the beam parameters which correspond to the two settings of the AGS-Booster, (set\#1 and set\#2) one may conclude on the dependence of the beam parameters $\left(\beta_{\mathrm{x}, \mathrm{y}}, \alpha_{\mathrm{x}, \mathrm{y}}\right)$ on the extraction settings of the AGS-Booster.

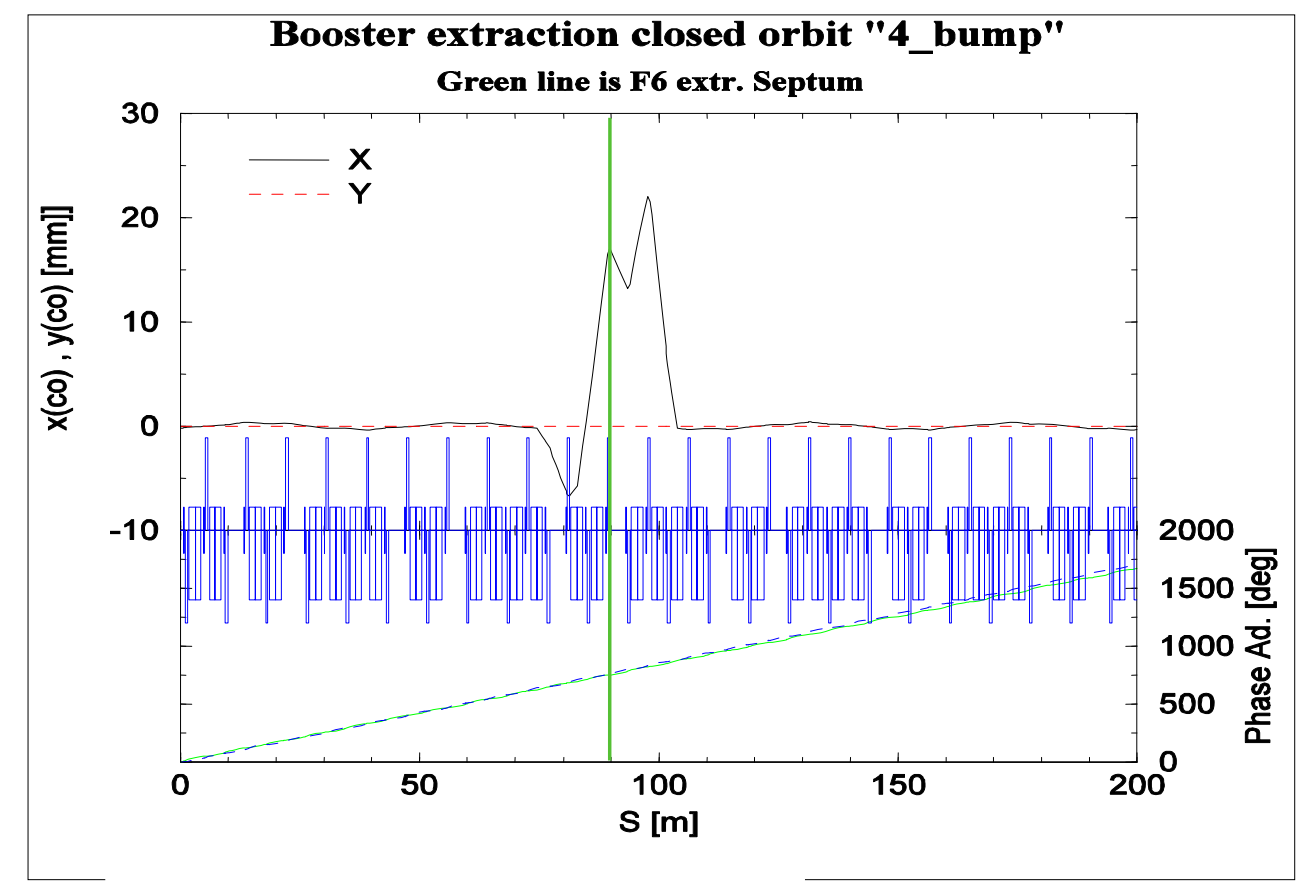

Figure 3. The magnets of the AGS-Booster (blue boxes) with the closed orbit displacement of the beam (black solid line) generated by the back leg windings (see text). The location of the F6 extraction septum is indicated by the green vertical line.

Table 1. Beam parameters at the Booster's extraction point for two extraction settings of the AGS-Booster. This Booster extraction point is also defined as the beginning of the BtA line.

\begin{tabular}{|l|l|l|l|l|l|l|l|l|}
\cline { 2 - 9 } \multicolumn{1}{c|}{} & Extraction setting & \multicolumn{6}{|c|}{ Beam parameters at Booster Extraction point } \\
\hline & Qx & Qy & $\beta_{\mathrm{x}}[\mathrm{m}]$ & $\alpha_{\mathrm{x}}$ & $\eta_{\mathrm{x}}[\mathrm{m}]$ & $\eta^{\prime}{ }_{\mathrm{x}}$ & $\beta_{\mathrm{y}}[\mathrm{m}]$ & $\alpha_{\mathrm{y}}$ \\
\hline Set\#1 & 4.679 & 4.803 & 4.797 & 0.819 & 1.606 & -0.415 & 10.890 & -1.676 \\
\hline Set\#2 & 4.789 & 4.773 & 4.530 & 0.836 & 1.282 & -0.561 & 10.982 & -1.691 \\
\hline
\end{tabular}

In this section we have described the procedure for the calculation of the beam parameters at the "beginning of the BtA" line. Before we proceed with the "BtA matching", we should calculate the beam parameters and dispersion functions at the "injection point of the AGS", which, in this technical note, is the middle of the straight section A06. In the following section we describe the procedure to calculate these beam parameters and the dispersion functions 


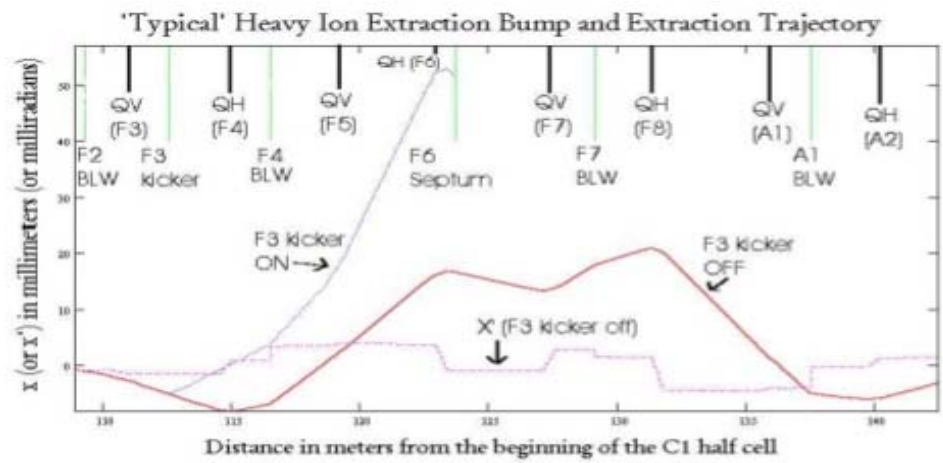

Figure 7.23: A model of the Booster extraction 4-bump in the extraction region. On the top of the graph the locations of various elements that determine the shape of the bump, and the F6 septum, are shown. The beam exits the machine at the F6 septum. Before the F3 kicker fires, the E.O. of an on-momentum particle follows the solid curve. The solid curve is the orbit distortion caused by the four backleg windings (at F2 (146.8 A), F4 (424.5 A), F7 (219.5 A), and A1 (595.2 A)). ( $\left.x, x^{\prime}\right)$ at F6 is (16.6 mm, $\left.-1.0 \mathrm{mrad}\right)$. When the F3 kicker is energized, or $O N$, the beam follows the extraction trajectory and exits at the F6 septum.

Figure 4. This figure has been borrowed from Ref. [2] and shows the location of the back leg windings which generate the beam bump, the location of the F3 extraction kicker and the location of the F6 septum.

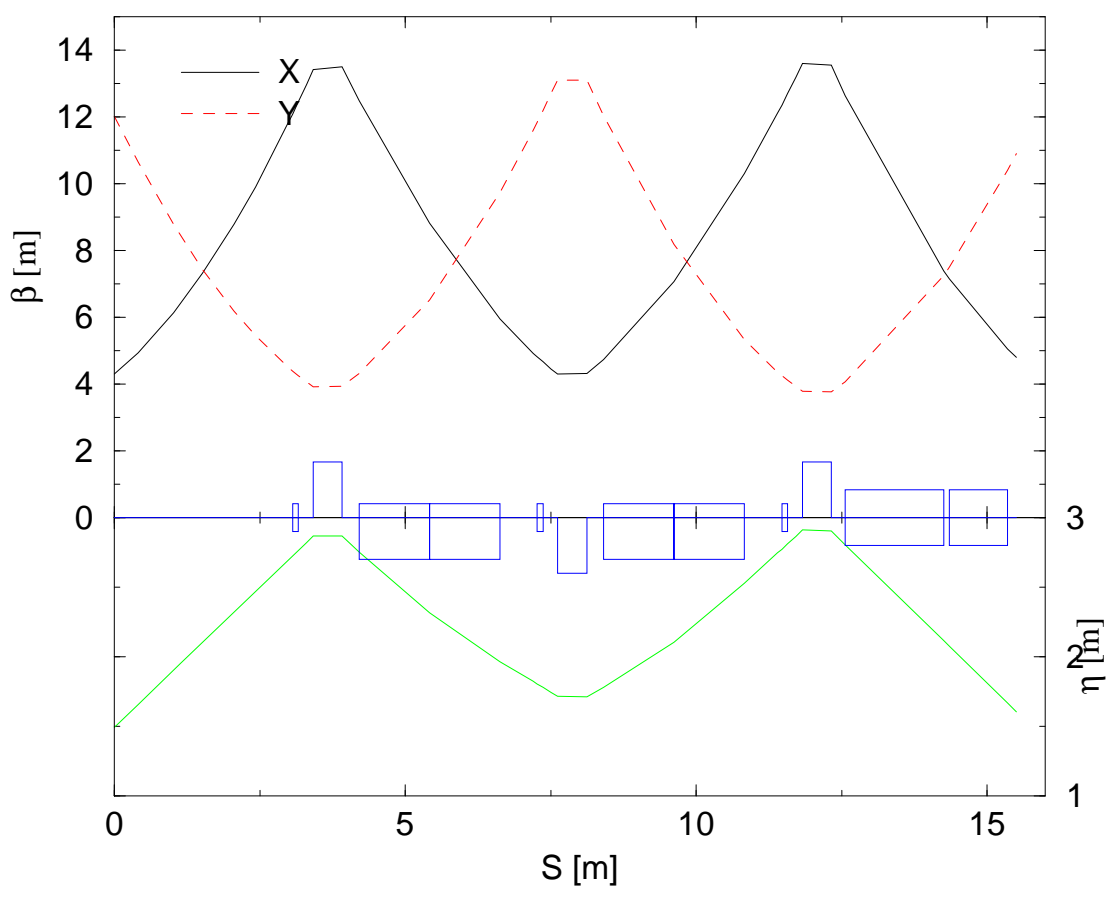

Figure 5. The beta functions (black and red lines) and horizontal dispersion function (green line) along the extraction section of the AGS-Booster. The last two blue boxes in 
the figure represent the F6 septum magnet which is comprised of two dipoles powered by the same power supply..

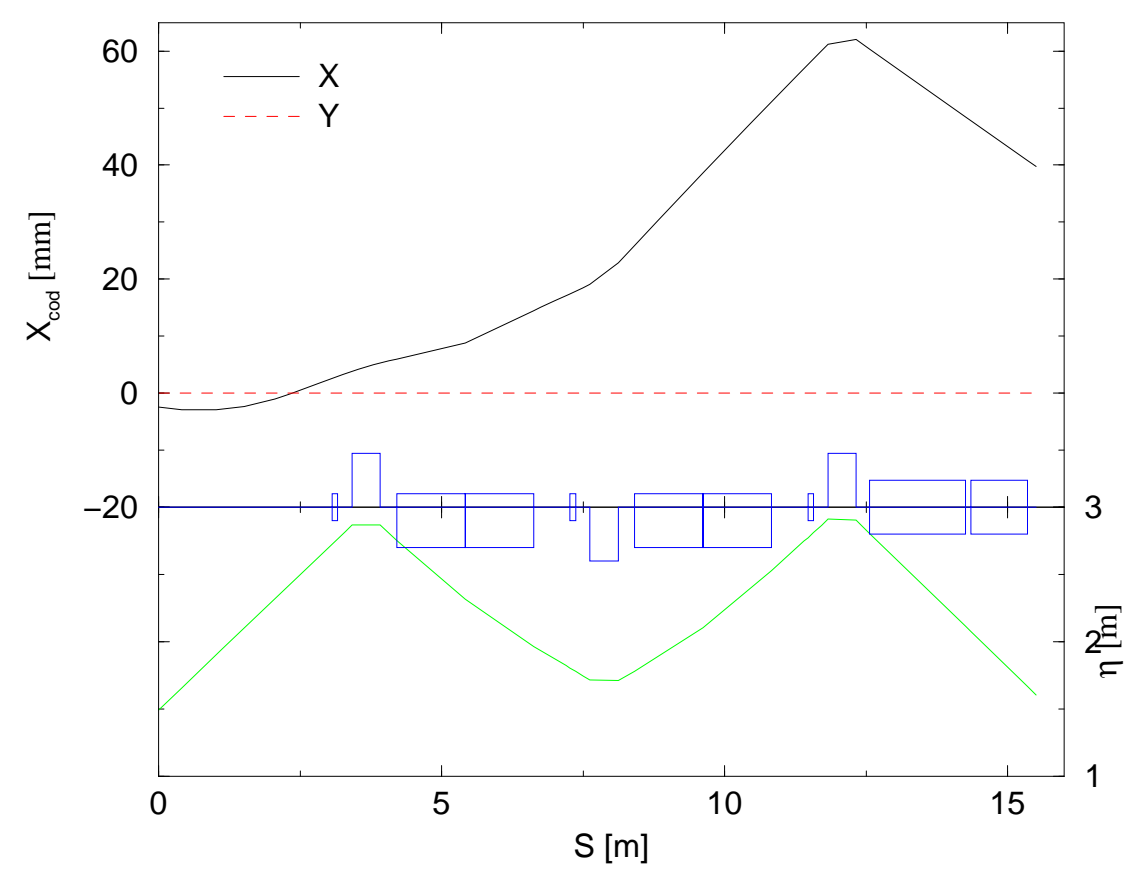

Figure 6. The trajectory of the extracted central orbit (black) along the extraction section of the AGS-Booster. The last two blue boxes in the figure represent the F6 septum magnet which is comprised of two dipoles connected in series and powered by a single power supply.

\section{Beam Parameters at the Injection Point of AGS}

This section is partitioned in two subsections; one subsection that describes the procedure to calculate the beam parameters of the circulating beam at the injection point of AGS using a MAD model of the AGS, and the other subsection, using a "local" model of the AGS which is referred as the BEAM++ model. This BEAM++ model of the AGS is included in a computer code BEAM++ [3] which originated in AGS and was further developed to include measured magnetic field maps of the main AGS magnets, as well as many other features, some of them described in Ref. [4,5].

\section{Calculation of Beam Parameters at the AGS injection point using a MAD Model for the AGS}

In the next subsection we discuss some of the features that the MAD model of the AGS uses.

\section{Some features of the MAD model of the AGS}

a) The combined function main magnets of the AGS incorporate the K1 and K2 values of the quadrupole and sextupole strengths, which are functions of the beam momentum. This functional dependence of the K1 and K2 quantities on 
the beam momentum has been introduced into the MAD model of the AGS a few years ago, but no information exists on this study.

b) In this MAD model, the combined function main magnets of the AGS, bend the circulating beam in the counter clockwise sense which is the actual sense of the circulating beam.

c) The effect of the L20 position and angle injection bump, which displaces the circulating beam near the L20 injection septum has been included in the calculations as follows:

c.1) The magnets that generate the L20 injection bump are made by a set of back leg windings placed on selected AGS main magnets. These back leg windings when excited generate a $3 / 2 \lambda$ position bump, and a $1 / 2 \lambda$ angle bump. Table 2 lists the main magnets with the back leg windings of the $\mathrm{L} 20$ position and angle bump.

c.2) The L20 position and angle bump, is modeled as a field error (dipole, quadrupole and sextupole) of a particular strength, that is imposed on each magnet with the back leg winding of the L20 beam bump. The strength of this field error is calculated by using fictitious kicker magnets at the center of the AGS main magnets which have the back leg windings. An optimization is performed on the L20 beam bump by adjusting the strength of these fictitious kicker magnets.

\section{AGS settings at Injection for a bare AGS}

a) Beam Rigidity at Injection $=8.372$ [T.m] $(\mathrm{p} / \mathrm{q}=2.51 \mathrm{GeV} / \mathrm{c})$

b) The Horizontal and Vertical tune Quads and the chromaticity sextupoles are not excited (Bare AGS).

c) The backleg windings which generate the "L20 position and angle" beam bump are excited and are modeled by Magnet-errors which are imposed on the corresponding main magnets of the AGS. The L20 beam bump displaces the circulating beam $\sim 45 \mathrm{~mm}$ at the center of the L20 straight section of AGS where the L20 injection septum is located.

Table 2. The list of the AGS main magnets with the back leg windings to generate the L20 beam bump. The numbers in parenthesis are the number of turns of each back leg winding.

\begin{tabular}{|l|l|}
\hline L20 position (3/2- $\lambda$ Bump) & K19(6), K20(6), L13(5), L14(5), A7(5), A8(5), B1(6), B2(6) \\
\hline L20 angle (1/2- $\lambda$ Bump) & L6(7), L7(7), A14(7), A15(7) \\
\hline
\end{tabular}

\section{Beam optics of the MAD-based model of the AGS at Injection}

Figure 7 shows the horizontal and vertical beta functions and the dispersion functions of the closed orbit of AGS at injection.

The black lines in Figure 8 show the horizontal beam displacement $\left(\mathrm{X}_{\mathrm{cod}}\right)$ and dispersion function $\left(\eta_{\mathrm{x}}\right)$ of the closed orbit in AGS at injection. The strength of the L20 position and angle bump which generates the beam bump shown in Fig. 8 is shown in the $2^{\text {nd }}$ row of 
Table 3. The location of the L20 injection septum is shown by the green vertical line in the figure.

The tunes, and chromaticities of the AGS at injection and the beam parameters, and the dispersion functions at the injection point of AGS (SS-A06) are presented in the $2^{\text {nd }}$ row of Table 4.

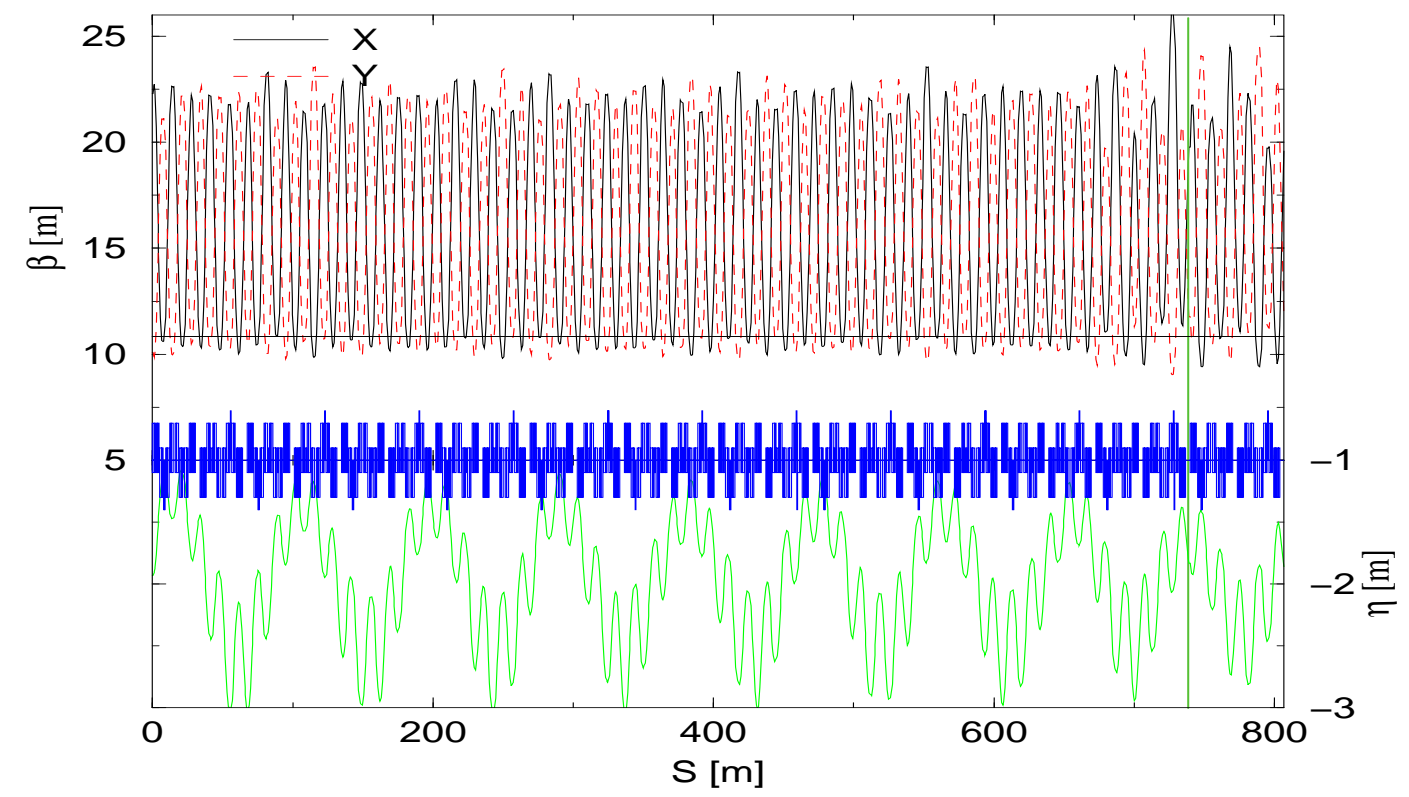

Figure 7. The horizontal (black line), vertical (red dashed line) beta functions and dispersion function (green line) at AGS injection as calculated by using a MAD based model of the AGS. The location of the L20 injection septum is shown by the vertical green line. The blue boxes represent the AGS main magnets.

Table 3. The strength of the L20 Position and Angle Bump.

\begin{tabular}{|c|c|c|c|c|}
\hline & $\begin{array}{c}\text { L20 Position } \\
{[\mathrm{mrad}]}\end{array}$ & $\begin{array}{c}\text { L20 Angle } \\
{[\mathrm{mrad}]}\end{array}$ & Xcod [mm] & X'cod [mrad] \\
\hline MAD & 0.67 & -0.55 & -44.6 & -2.27 \\
\hline BEAM++ & 0.72 & -0.50 & -47.5 & -1.25 \\
\hline
\end{tabular}

Table 4. Tunes, chromaticities of AGS and beam parameters and dispersion functions at the Injection point of AGS (SS-A06).

\begin{tabular}{|c|c|c|c|c|c|c|c|c|c|c|}
\hline & $\mathrm{Q}_{\mathrm{x}}$ & $\mathrm{Q}_{\mathrm{y}}$ & $\xi_{x}$ & $\xi_{\mathrm{y}}$ & $\begin{array}{c}\beta_{\mathrm{x}} \\
{[\mathrm{m}]}\end{array}$ & $\alpha_{x}$ & $\begin{array}{c}\eta_{\mathrm{x}} \\
{[\mathrm{m}]}\end{array}$ & $\eta_{x}^{\prime}$ & $\begin{array}{c}\beta_{\mathrm{y}} \\
{[\mathrm{m}]}\end{array}$ & $\alpha_{y}$ \\
\hline MAD & 8.711 & 8.772 & -22.7 & 1.7 & 16.2 & 2.01 & -2.08 & 0.204 & 15.4 & -1.91 \\
\hline BEAM++ & 8.751 & 8.784 & -11.6 & -8.9 & 14.4 & 1.69 & -1.94 & 0.209 & 16.0 & -1.80 \\
\hline MAD only & 8.711 & 8.772 & 22.7 & 1.7 & 16.3 & 2.01 & -2.01 & 0.204 & 15.3 & -1.90 \\
\hline
\end{tabular}




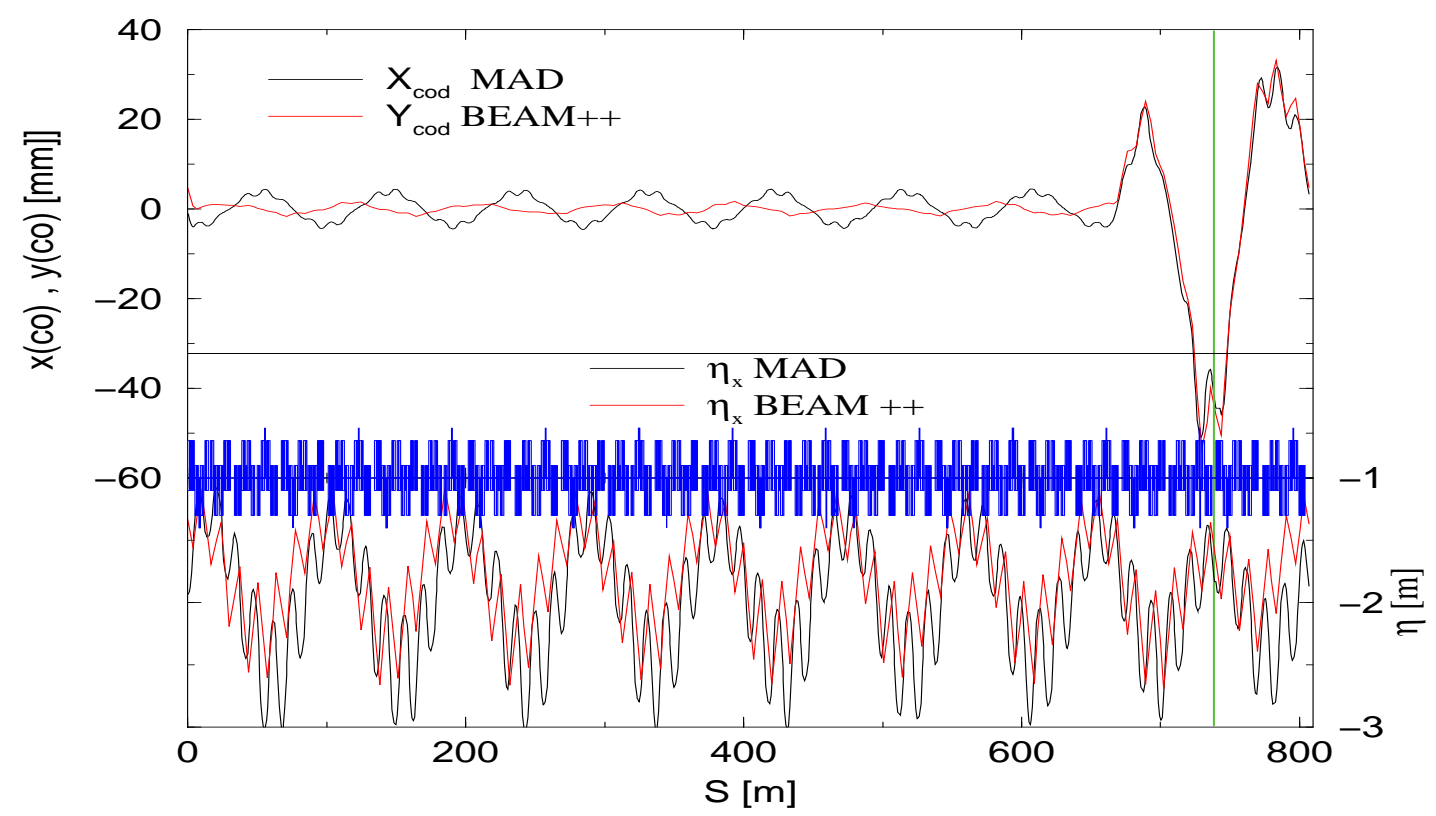

Figure 8. The horizontal (black line) beam displacement $\left(\mathrm{X}_{\mathrm{cod}}\right)$ and dispersion function $\left(\eta_{\mathrm{x}}\right)$ of AGS at injection, as calculated by using a MAD-based model of the AGS. The read lines correspond to the same quantities $\left(\mathrm{X}_{\mathrm{cod}}\right)$ and $\left(\eta_{\mathrm{x}}\right)$ as calculated using the BEAM++ model of the AGS. The location of the L20 injection septum is shown by the vertical green line. The blue boxes represent the AGS main magnets.

\section{Calculations of Beam Parameters at the Injection point of the AGS using the BEAM++ Model of the AGS}

This method of calculating the beam parameters and the dispersion functions of the beam's closed orbit is equivalent to the one used in the MAD based model of the AGS. In this method the BEAM++ code ray traces the particle on the central orbit in measured magnetic field maps [4,5] and it calculates the beam parameters and dispersion functions along the AGS ring. The L20 injection bump mentioned in the previous section has also been introduced in the BEAM++ version. Details on the BEAM++ code and on raytracing in experimental field maps appear in Ref. [ 4].

As in the MAD based model, the AGS has been set at a Beam Rigidity $=8.372$ [T.m] $(\mathrm{p} / \mathrm{q}=2.51 \mathrm{GeV} / \mathrm{c})$ and with the Horizontal and Vertical tune Quads and chromaticity sextupoles not excited (Bare AGS).

\section{Beam optics of the AGS at Injection based in the BEAM++ model}

Figure 9 shows the horizontal and vertical beta functions and the dispersion function of the closed orbit of AGS at injection.

The red lines in Figure 8 show the horizontal beam displacement $\left(\mathrm{X}_{\text {cod }}\right)$ and dispersion function $\left(\eta_{\mathrm{x}}\right)$ of the closed orbit in AGS at injection. The strength of the L20 position and angle bump which generated the beam bump shown in Fig. 8 is shown in the $3^{\text {rd }}$ column 
of Table 3. The location of the L20 injection septum is shown by the green vertical line in figure 8.

The tunes, and chromaticities of the AGS at injection and the beam parameters, and the dispersion functions at the injection point of AGS (SS-A06) are presented in $3^{\text {rd }}$ row of Table 4.

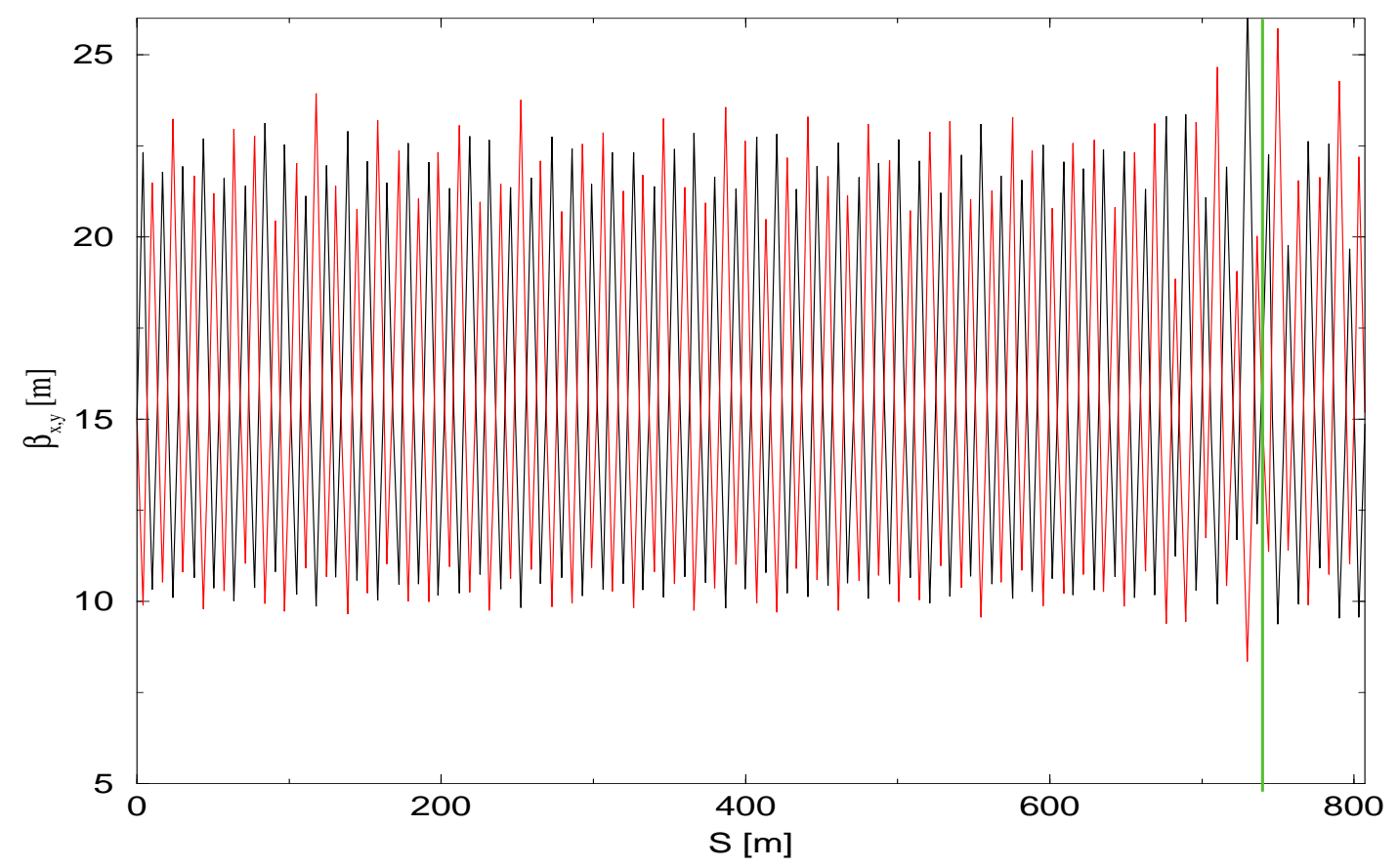

Figure 9. The horizontal (black line), vertical (red dashed line) beta functions as calculated by using the BEAM++ based model of the AGS. The location of the L20 injection septum is shown by the vertical green line. This figure is good to show the maximum and minimum variations of the beta functions along the AGS ring.

\section{Calculation of the $R$ matrix between the end point of the BtA line and the middle of the straight section A06 of AGS (Inj. point)}

In order to proceed with the "BtA matching", we need to know the R matrices of the magnetic elements of the "AGS injection section" which is defined between the end of the BtA line (28.6 cm from the exit of the QV15 quadrupole) and the AGS injection point A06. This "AGS injection section" starts from the end of the BtA line, continues in the fringe field of the M240 main magnet to the L20 injection septum, with the rest of the line consisting of the AGS lump magnetic elements (from L20 septum to the AGS injection point). In the following sections we are presenting results of the BtA transport using either the R_matrix or the lump elements representation of the AGS injection section between the L20 septum and the AGS injection point.

Some details on the calculations of the $R$ matrix between the end of the BtA line and the injection point (SS A06) of AGS

Establish the end point of the BtA line and the beam direction at this point 
Since the method we are using to calculate the $\mathrm{R}$ matrix is based on raytracing, we need to know the location of the end point of the BtA line and the direction of the beam at this point, in relation to the location of the AGS magnets. The location of the end point of the BtA line and the direction of the beam at this point relative to the AGS is presented in the schematic diagram of Fig. 10. The "point 3" in Fig. 10 which represents the end of the BtA line has been calculated as follows:

a) We use the Survey points [6] Sp\#1 to Sp\#4 shown in Fig. 10 and Table 5 to calculate the equations of the following two straight lines

a1) the line SS-239 defined by the points Sp\#1 and Sp\#2 and

a2) the line of the last part of the BtA line defined by the points Sp\#4 and Sp\#3.

b) We calculate the distance of the BtA line from the "point 1 " which is the middle of the SS-239. This distance is between "point 1" and "point 2" shown in the Fig. 10 and is equal to 569.7 [mm].

c) To define the end of the BtA line, we move from "point 2" a distance $\Delta \mathrm{s}=60 \mathrm{~cm}$ (downstream along the BtA line which makes and angle of $X^{\prime}=-139.39$ [mrad] with the SS-239) to "point 3" which is the end of the BtA line.

Table 5. The survey coordinates of the point appearing in Fig. 10

\begin{tabular}{|l|l|l|}
\hline Survey point & North [inches] & East [inches] \\
\hline Sp \#1 & 13470.3532 & 2104.1939 \\
\hline Sp \#2 & 13437.728 & 2094.6247 \\
\hline Sp \#3 & 13458.813 & 2078.35623 \\
\hline Sp \#4 & 13556.3479 & 2092.6908 \\
\hline
\end{tabular}

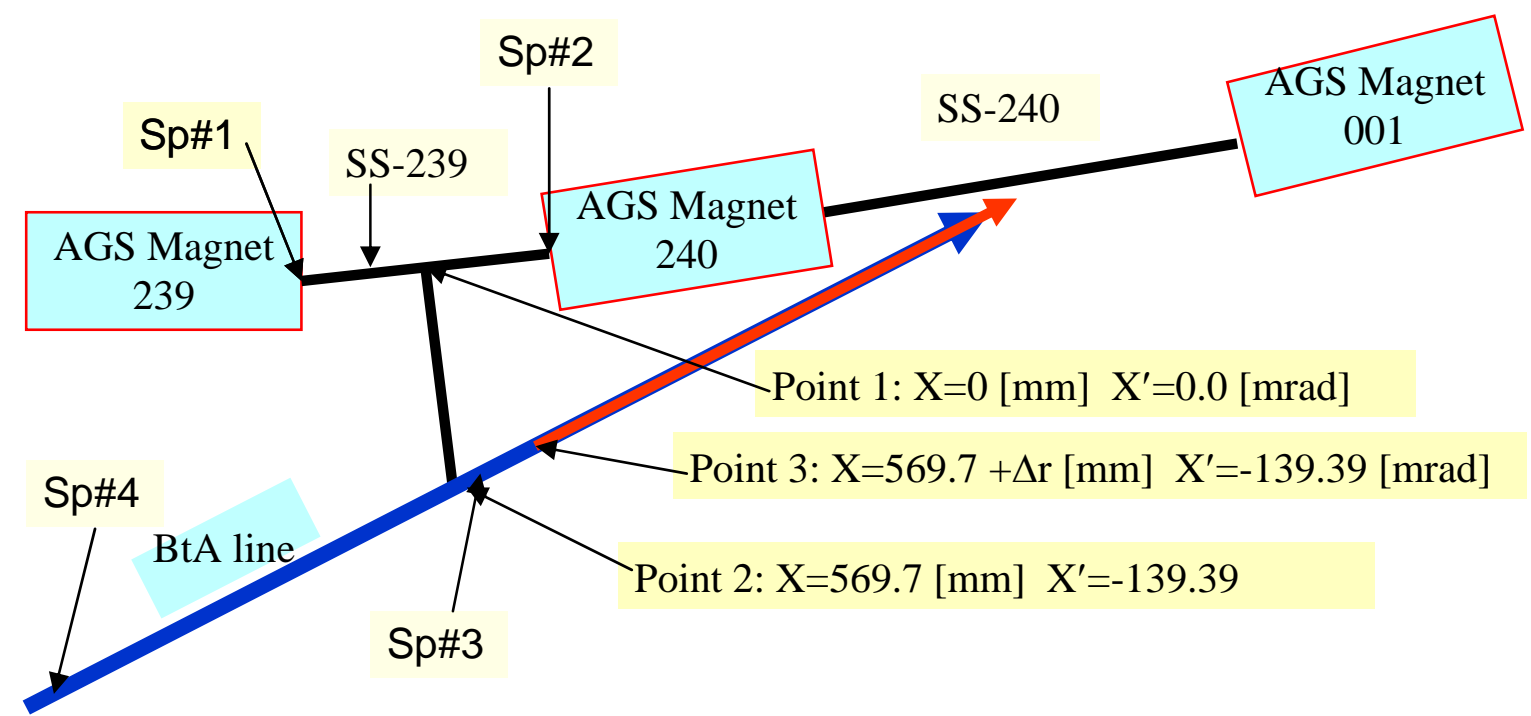

Figure 10. Schematic diagram of the BtA line in relation to the AGS. The survey points (Sp) shown in this figure, and in Table 5, are used to establish the "end point" of the BtA line and the beam direction at this point.

\section{Calculation of the R-matrix between the end of the BtA line and the injection point in AGS}

Starting from end of the BtA line, ("point 3" in Fig. 10) we raytrace the central particle to the injection point of AGS (SS-A06). The strengths of the L20 septum and the A05 
kicker of the AGS are adjusted for the injected beam to be collinear with the circulating beam at the exit of the A05 kicker. The BEAM++ code which raytraces the central particle, calculates also the matrix elements $\mathrm{R}_{\mathrm{ij}}\{\mathrm{i}=1$ to $6, j=1$ to 6$\}$ of the $6 \times 6 \mathrm{R}$-matrix which appear below.

$\begin{array}{llllll}-0.518630 & 13.971178 & 0.0 & 0.0 & 0.0 & 0.341300 \\ -0.011256 & -1.624946 & 0.0 & 0.0 & 0.0 & -0.131200 \\ 0.0 & 0.0 & 0.919300 & 17.557420 & 0.0 & 0.0 \\ 0.0 & 0.0 & 0.045918 & 1.9647490 & 0.0 & 0.0 \\ -0.071886 & 1.278425 & 0.0 & 0.0 & 1.0 & 0.0 \\ 0.0 & 0.0 & 0.0 & 0.0 & 0.0 & 1.0\end{array}$

\section{Matching of the BtA line to the middle of the straight section A06 (AGS Injection point). The Beam Transport $R$ matrix from the exit of BtA line to SS_A06 is calculated with BEAM++ code}

In this section we present the matching of the BtA line to the injection point of the AGS. For the matching we are using the following information:

a) The MAD model of the BtA line.

b) The beam parameters at the beginning of the BtA line (Booster Extraction point), which were calculated in an earlier section.

c) The R matrix between the end of the BtA line and the AGS injection point (see previous section). This $\mathrm{R}$ matrix was calculated using the BEAM++ code as discussed in the previous section.

In the next section we present a different approach for the transport of the injected beam between the L20 septum and the A06 straight section. In this different approach, we use the lump magnetic elements of the injection section, as provided by the MAD model of the AGS.

d) The beam parameters at the injection point of the AGS which were calculated in an earlier section, using either, the BEAM++ model, or the MAD model of the AGS.

No constraints, other than that the beam should fit in the vacuum pipe, are imposed along the BtA line.

Figure 11 shows the beta functions and the dispersion functions of the BtA line which matches the beam parameters of the injected beam to those of the circulating beam (as calculated by the BEAM++ code) at the injection point of AGS. The values of the beam parameters and the dispersion functions at the matching point (AGS injection point) appear in the third row of Table 4 . The K1 values of the quadrupoles of the BtA line appear in the $3^{\text {rd }}$ column of Table 6. 


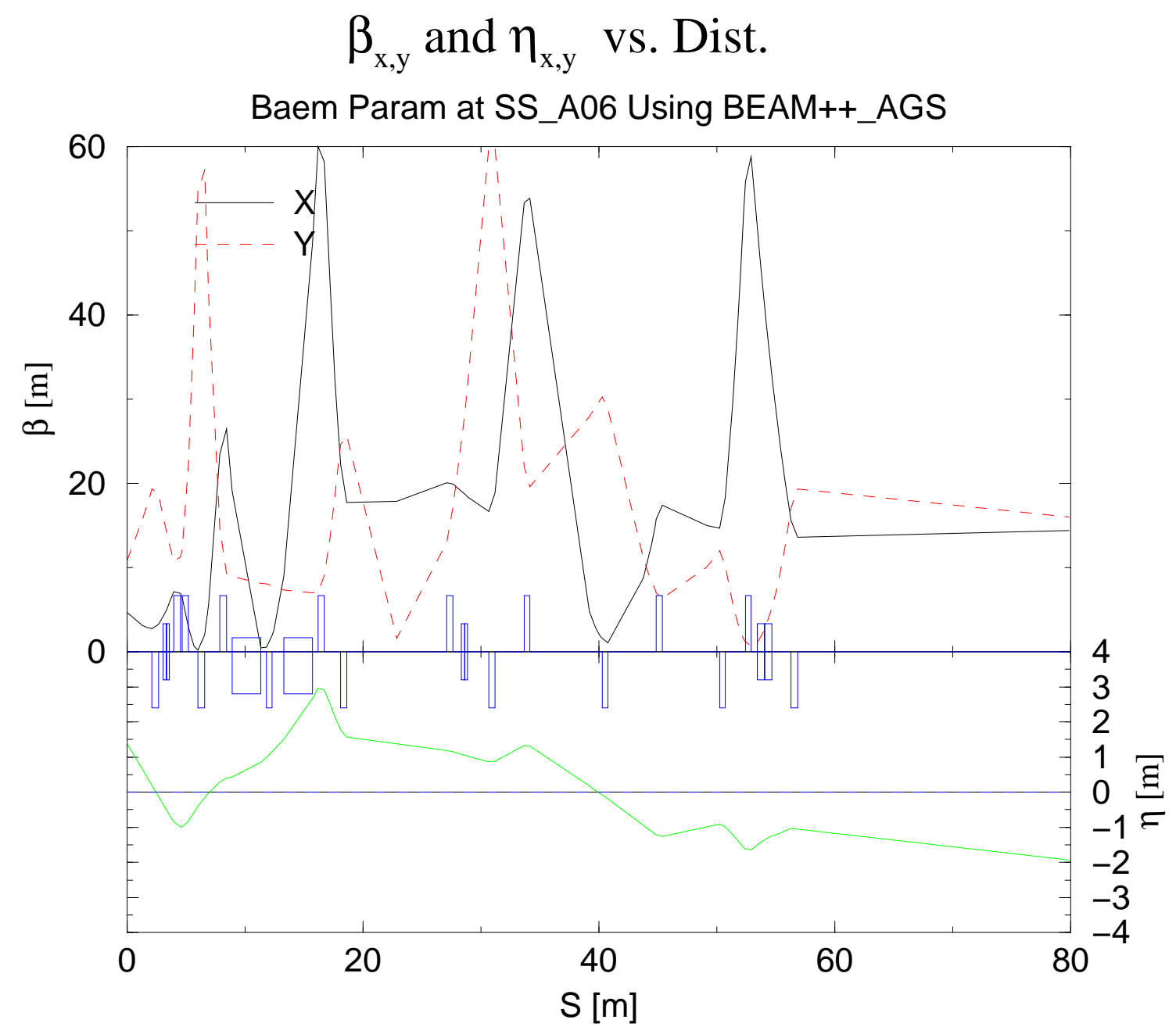

Figure 11. The horizontal and vertical beta functions and the dispersion function along the BtA line which matches the injected beam to the circulating beam of AGS at the AGS injection point. The beam parameters at the AGS injection point were calculated using the $\mathrm{BEAB}++$ computer code.

Similarly Figure 12 shows the beta functions and the dispersion functions of the BtA line which matches the beam parameters of the injected beam to those of the circulating beam (as calculated by the MAD code) at the injection point of AGS. The values of the beam parameters and the dispersion functions at the matching point (AGS injection point) appear in the second row of Table 4 . The K1 values of the quadrupoles of the BtA line appear in the $2^{\text {rd }}$ column of Table 6. 


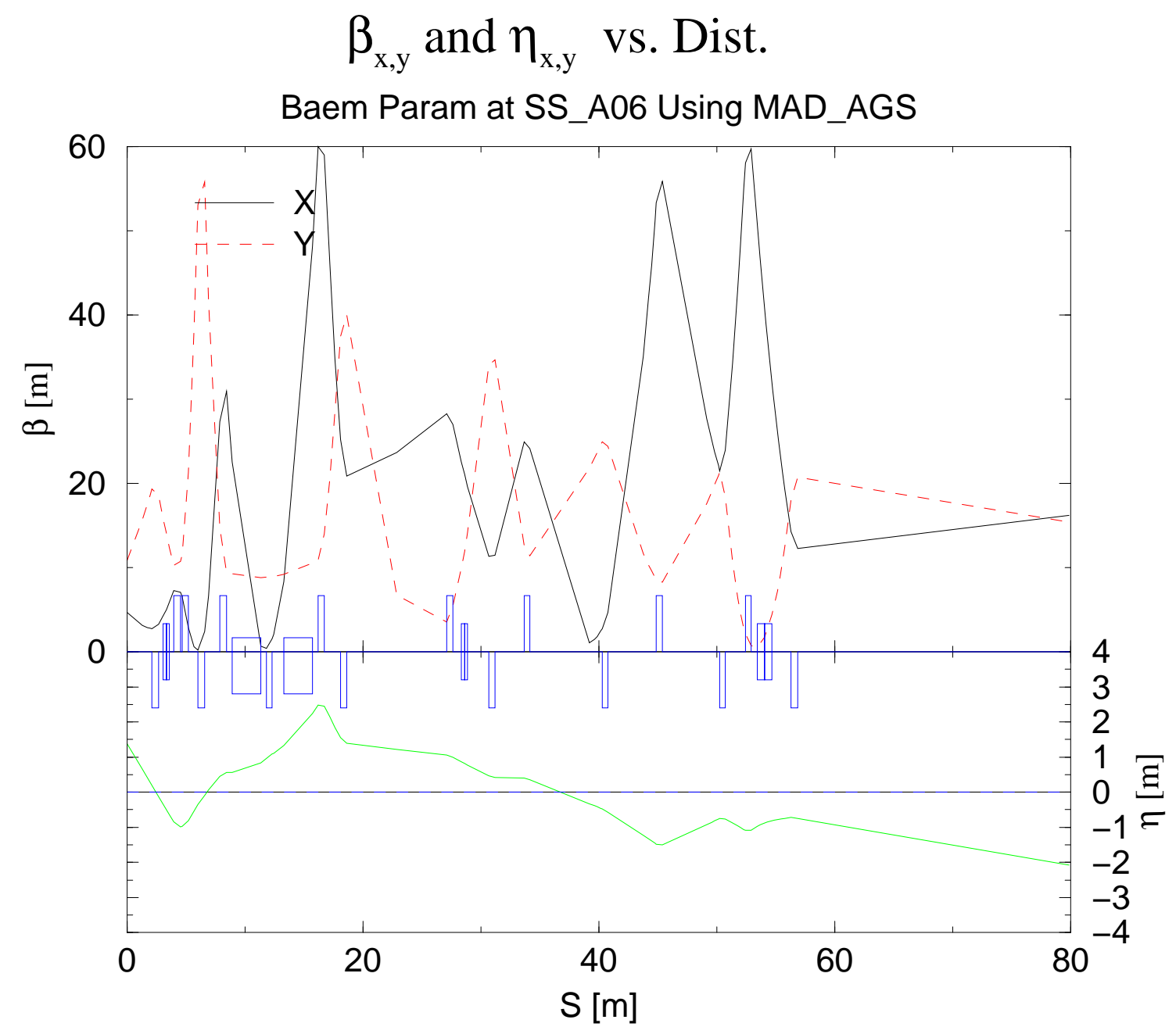

Figure 12. The horizontal and vertical beta functions and the dispersion function along the BtA line which matches the injected beam to the circulating beam of AGS. The beam parameters at the AGS injection point were calculated using the MAD based model of the AGS.

In either beam transport which is generated by the $\mathrm{K} 1$ values appearing in $2^{\text {nd }}$ and $3^{\text {rd }}$ columns of Table 6, the polarity for the BtA quadrupoles was kept the same as it appears in the "BtA magnet application" of the main control room (Nominal polarity). It is possible however to reduce the maximum value of the beta function along the BtA line, if we remove this constrain of using the "Nominal polarity" of the BtA quadrupoles.

Table 6. Three sets of the $\mathrm{K} 1$ values of the BtA quadrupoles which can match the beam parameters of the injected beam, to those of the circulating beam in AGS. The values in column 2 and 3 correspond to the "BtA beam matching" to the beam parameters as calculated by the a) BEAM++ code $\left(2^{\text {nd }}\right.$ column $)$ b) the MAD based AGS model ( $3^{\text {rd }}$ column). and c) the MAD based modeled AGS ( $4^{\text {th }}$ column) but using lump magnetic elements for the AGS injection section between the L20 to the AGS Injection point. 


\begin{tabular}{|c|c|c|c|}
\hline & $\begin{array}{c}\left.\mathrm{K} 1 \mathrm{~m}^{-2}\right] \\
\{\text { BEAM++ code }\}\end{array}$ & $\begin{array}{c}\left.\mathrm{K} 1 \mathrm{~mm}^{-2}\right] \\
\{\mathrm{MAD} \text { AGS Model }\}\end{array}$ & $\begin{array}{c}\mathrm{K} 1\left[\mathrm{~m}^{-2}\right] \\
\{\mathrm{MAD} \text { AGS ONLY }\}\end{array}$ \\
\hline Q1 & $-5.489035 \mathrm{E}-01$ & $-5.667196 \mathrm{E}-01$ & $-5.957579 \mathrm{E}-01$ \\
\hline Q2 & $1.032342 \mathrm{E}+00$ & $1.065251 \mathrm{E}+00$ & $9.714537 \mathrm{E}-01$ \\
\hline Q3 & $-1.440037 \mathrm{E}+00$ & $1.440671 \mathrm{E}+00$ & $-1.408778 \mathrm{E}+00$ \\
\hline Q4 & $1.461645 \mathrm{E}+00$ & $1.434935 \mathrm{E}+00$ & $1.472116 \mathrm{E}+00$ \\
\hline Q5 & $-9.913408 \mathrm{E}-02$ & $-5.627279 \mathrm{E}-03$ & $-2.586744 \mathrm{E}-02$ \\
\hline Q6 & $9.412080 \mathrm{E}-01$ & $9.043740 \mathrm{E}-01$ & $8.620935 \mathrm{E}-01$ \\
\hline Q7 & $-9.326969 \mathrm{E}-01$ & $-8.251799 \mathrm{E}-01$ & $-8.094347 \mathrm{E}-01$ \\
\hline Q8 & $1.052036 \mathrm{E}-01$ & $2.793435 \mathrm{E}-01$ & $3.043382 \mathrm{E}-01$ \\
\hline Q9 & $-6.172236 \mathrm{E}-01$ & $-7.119555 \mathrm{E}-01$ & $-6.364001 \mathrm{E}-01$ \\
\hline Q10 & $6.069770 \mathrm{E}-01$ & $6.591937 \mathrm{E}-01$ & $5.106957 \mathrm{E}-01$ \\
\hline Q11 & $-3.281473 \mathrm{E}-01$ & $-3.328328 \mathrm{E}-01$ & $-4.420591 \mathrm{E}-01$ \\
\hline Q12 & $5.146911 \mathrm{E}-01$ & $5.013254 \mathrm{E}-01$ & $4.581065 \mathrm{E}-01$ \\
\hline Q13 & $-9.613420 \mathrm{E}-01$ & $-9.332006 \mathrm{E}-01$ & $-3.329547 \mathrm{E}-01$ \\
\hline Q14 & $7.851872 \mathrm{E}-01$ & $7.230360 \mathrm{E}-01$ & $4.783211 \mathrm{E}-01$ \\
\hline Q15 & $-5.834841 \mathrm{E}-01$ & $-6.597904 \mathrm{E}-01$ & $-3.636073 \mathrm{E}-01$ \\
\hline
\end{tabular}

\section{"Matching" the BtA line to bare AGS. The beam transport from the exit of the BtA line to the AGS injection point is calculated using a MAD model of the AGS}

This section is similar to the previous section with the only difference being in the items c) and d) below.

For the matching we are using the following information:

a) The MAD model of the BtA line.

b) The beam parameters at the beginning of the BtA line (Booster Extraction point), which were calculated in an earlier section.

c) The beam transport of the injected beam between the L20 septum and the AGS injection point (SS_A06) is using the magnetic elements of the AGS, as are used in the MAD model of the AGS. This is the main difference of the previous section, with this section, which provides the values of the beta functions and dispersion functions at the entrance and exit of the various elements of the section.

d) The beam parameters at the injection point of the AGS which were recalculated using a MAD model of the AGS, as we have done earlier, with the only difference we bumped the circulating beam in the AGS at the L20 septum by $30 \mathrm{~mm}$ instead of $45 \mathrm{~mm}$.

e) In this section we also use an R_matrix to describe the beam transport from the exit of the BtA line to the entrance of the L20 septum. This R_matrix is calculated using the BEAM++ code and contains the effect on the beam, of the fringe field produced by the L240 AGS magnet.

For the beam matching, no constraints, other than that the beam should fit in the vacuum pipe, are imposed along the BtA line. 
Figure 13 shows the beta functions and the dispersion functions of the BtA line which matches the beam parameters of the injected beam to those of the circulating beam (as calculated by the MAD model of the AGS) at the injection point of AGS. This figure 13 shows the values of the beta functions at the beginning and the exit of the magnetic elements of the section of the AGS between SS_L20 and SS_A06. The values of the beam parameters and the dispersion functions at the matching point (AGS injection point) appear in the last row of Table 4. The K1 values of the quadrupoles of the BtA line appear in fourth column Table 6.

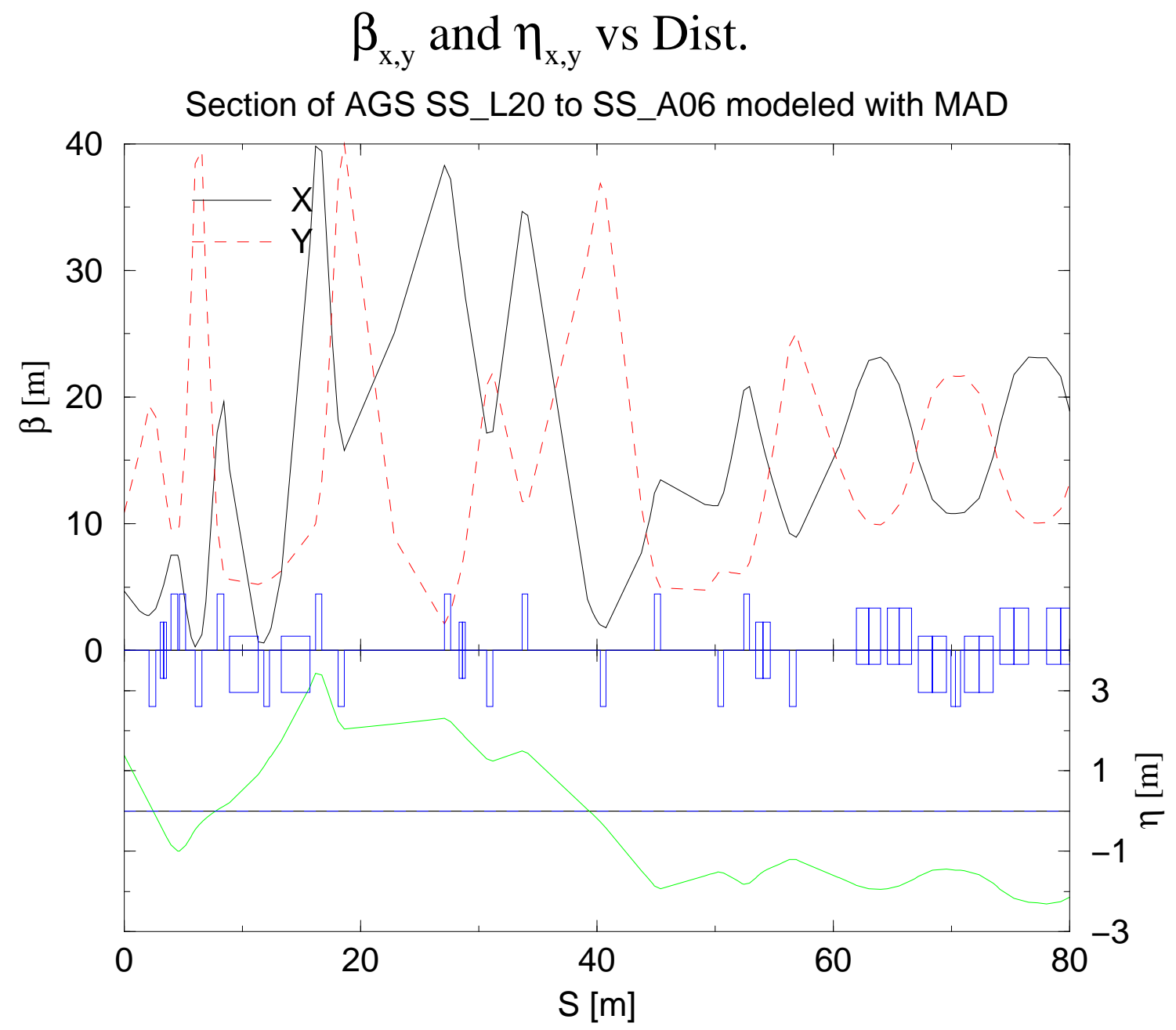

Figure 13. The horizontal and vertical beta functions and the dispersion function along the BtA line and the AGS section from the SS_L20 to SS_A06. The BtA line matches the injected beam to the circulating beam of AGS. The beam parameters at the AGS injection point as well as along the AGS injection section, (SS_L20 to SS_A06) were calculated using the MAD based model of the AGS.

The horizontal beam displacement $\left(\mathrm{X}_{\mathrm{cod}}\right)$ of the circulating beam and that of the injected beam is shown in Figure 14. Note that the $\mathrm{X}_{\text {cod }}$ of both beams merge into one after the A05 kicker. 


\section{Xcod and $\eta_{x, y}$ vs Dist.}

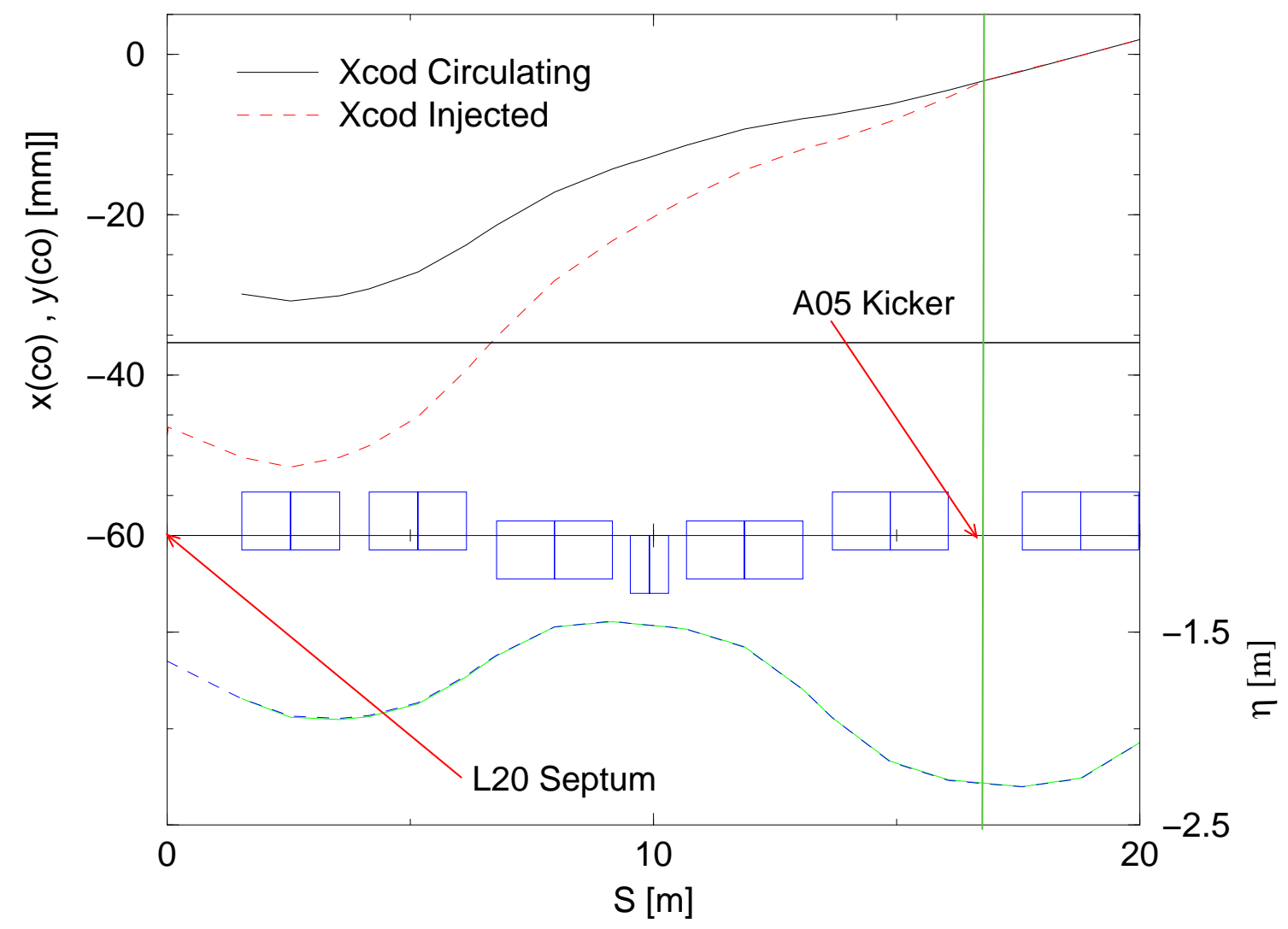

Figure 14. A section of the closed orbit displacements ( $\left.\mathrm{X}_{\mathrm{cod}}\right)$ in AGS (black line) and of the injected beam (read dashed line). The location of the L20 septum and of the A05 kicker are shown by red arrows. Note that the L20 septum and A05 kicker are "kicking the injected beam to aligning it with the circulating beam at the exit of the A05 kicker.

The elements of the R_matrix which corresponds to the beam transport of the AGS Injection section (L20 to A06) are shown below. These matrix elements which are calculated using a MAD based model of the AGS are to be compared with those which were calculated by raytracing using the BEAM++ code, and appear in an earlier section.

$\begin{array}{lllllc}-0.91682 & 15.11163 & 0.0 & 0.0 & 0.0 & 1.027 \\ 0.02689 & -1.53393 & 0.0 & 0.0 & 0.0 & -0.202 \\ 0.0 & 0.0 & 1.30159 & 14.98233 & 0.0 & 0.0 \\ 0.0 & 0.0 & 0.09737 & 1.88911 & 0.0 & 0.0 \\ -0.157582 & 1.477203 & 0.0 & 0.000000 & 1.0 & 0.0 \\ 0.0 & 0.0 & 0.0 & 0.0 & 0.0 & 1.0\end{array}$




\section{Sensitivity studies for the BtA line}

In this section we present results on the dependence of the beam parameters at the AGS injection point on the random variations of the magnet strengths of the BtA line.

In this sensitivity study we have assumed that the strength of the quadrupole magnets can vary by $\pm 0.2 \%$, and the strength of the BtA dipole magnets by $\pm 0.1 \%$ from their nominal value. These errors are uniformly distributed between these upper and lower limits.

The effect on the beam from the error on the L20 septum is considered separately and appears in Table 7 which summarizes this sensitivity study. We have assumed no error in the A05 kicker magnet.

Table 7 summarizes the results of from fifty random variations in the strength of the BtA magnets. Each random variations in the strength of a magnet is randomly selected from a set of numbers uniformly distributed in the range $\pm 0.2 \%$ for quadrupoles , and $\pm 0.1 \%$, for dipoles.

In Table 7 the $3^{\text {rd }}$ row shows the Nominal values of the beam parameters and the horizontal dispersion functions, at the injection point (SS_A06) of the AGS. The $2^{\text {nd }}$ and $4^{\text {th }}$ rows shows the maximum and minimum values respectively, that a particular quantity can obtain under a particular set of random variations in the strength of the BtA magnets. The $5^{\text {th }}$ and $6^{\text {th }}$ rows show the average value and the standard deviation of each physical quantity as calculated from a set of fifty values.

Table 7. Beam parameters at the injection point as affected by the random variations of the BtA magnets (See text).

\begin{tabular}{|l|c|c|c|c|c|c|c|c|}
\hline & $\beta_{\mathrm{x}}[\mathrm{m}]$ & $\alpha_{\mathrm{x}}$ & $\mathrm{X}[\mathrm{mm}]$ & $\mathrm{XP}[\mathrm{mrad}]$ & $\eta_{\mathrm{x}}[\mathrm{m}]$ & $\eta_{\mathrm{x}}^{\prime}$ & $\beta_{\mathrm{y}}[\mathrm{m}]$ & $\alpha_{\mathrm{y}}$ \\
\hline Max & 17.160 & 2.112 & 9.632 & 2.746 & -2.008 & 0.285 & 17.358 & -1.735 \\
\hline Nominal & 16.190 & 2.017 & 2.410 & 1.589 & -2.008 & 0.209 & 15.510 & -1.917 \\
\hline Min & 15.523 & 1.929 & -5.207 & 0.489 & -2.467 & 0.209 & 14.244 & -2.176 \\
\hline Average & 16.295 & 2.027 & 2.169 & 1.617 & -2.349 & 0.267 & 15.435 & -1.908 \\
\hline Stand.dev. & 0.058 & 0.006 & 0.403 & 0.062 & 0.010 & 0.002 & 0.093 & 0.013 \\
\hline & & & & & & & & \\
\hline
\end{tabular}

A variation by $\pm 0.1 \%$ of the L20 septum affects the position and the angle of the injected central trajectory by \pm 2.5 [mm] and \pm 0.25 [mrad] respectively at the injection point SSA05. The rest of the beam parameters and dispersion functions at the injection point, remain virtually unchanged.

\section{Conclusions}

In this technical note we have shown that the BtA line has adequate variables to match the transported beam from the AGS-Booster to the circulating beam of a bare AGS. A sensitivity study of the BtA line shows that variations in the strength of the power supplies of the BtA line by $\pm 0.2 \%$ in the dipoles and $\pm 0.1 \%$ in the quadrupoles maybe tolerated. This study also shows that the MAD-based model of the AGS differs from that of the BEAM++ model as one can conclude from the comparison of the calculated tunes 
and chromaticities which appear in Table 4. An experimental study is required to help resolve this disagreement. In a following brief technical note (Part II) we will show that the BtA line can also match the beam parameters and dispersion functions of the injected beam to those of the circulating beam of an AGS which is equipped with two helical snakes.

\section{References}

[1] Reference for the BtA line.

[2] K. Zeno, A Rookie's Guide to Booster Operations, Booster Tech. Note \#231, Sept. 29, 1998

[3] E.D. Courant “The AGS Orbit Computing Program” AGS Internal Report.

[4] N. Tsoupas et. al. "Closed Orbit Calculations at AGS, and Extraction Beam

Parameters at H13" BNL-AGS Tech. Note AD/RHIC/RD-75.

[5] N. Tsoupas et. al. "R-matrices of the Fast Extraaction Beam (FEB) of the AGS, and Beam Parameters at the Starting Point of AtR" BNL-AGS Tech. Note C-A/AP/300

[6] F. Karl private communication. 